\title{
Characterizing the epidemiology and interaction between HIV-1 and HBV co-infection in South Africa
}

\author{
Philippa C Matthews ${ }^{1,2^{*}}$, Apostolos Beloukas ${ }^{3}$, Amna Malik ${ }^{4}$, Thumbi Ndung' $u^{5}$, Philip Goulder ${ }^{4,5}$, \\ Anna Maria Geretti ${ }^{3}$, Paul Klenerman ${ }^{1,2,6}$ \\ From International Symposium HIV and Emerging Infectious Diseases 2014 \\ Marseille, France. 21-23 May 2013
}

\section{Introduction}

Anti-Retroviral Therapy (ART) has dramatically reduced morbidity and mortality associated with HIV/AIDS. However, this has left a niche for the emergence of liver disease in HIV-positive individuals co-infected with HBV. Despite the geographical overlap between highly endemic HBV and HIV in Southern Africa, there is a wide range in the prevalence of co-infection. We therefore set out to characterize the epidemiology of HIV/HBV co-infection in a Durban cohort, and to investigate the possible impact of HBV infection on HIV disease progression.

\section{Materials and methods}

We investigated a cohort of 498 adult women recruited via antenatal/postnatal clinics in Durban, South Africa, of whom 72 were HIV negative and 426 were chronically HIV-infected and ART-naïve (median CD4 count 368 cells/mm3, median HIV-1 RNA load $4.47 \log 10$ copies/ $\mathrm{ml}$ ). We screened plasma for HBsAg by ELISA (Biokit). CD8+ T cell responses to HIV peptides were quantified by IFN-gamma ELISpot assay in $325 \mathrm{HIV}$-infected individuals including 35 with HBV coinfection.

\section{Results}

Overall HBsAg prevalence was 46/498 (9.2\%; 95\%-confidence interval 7-12\%); coinfection rates were $9.4 \%$ in HIVpositive and $8.3 \%$ in HIV-negative individuals. CD4 counts were significantly lower in with HBV/HIV coinfection than with HIV monoinfection (302 vs. 375 cells/mm3; $\mathrm{p}=0.02)$. However, HBV status made no significant impact on HIV viral load (4.49 $\log 10$ copies/ml in coinfection vs. $4.46 \log 10$ in monoinfection). There was no difference in breadth, magnitude, or protein-specificity of IFN-gamma responses to HIV according to HBV status.

\section{Conclusions}

In this cohort of Durban women, 9\% were coinfected with HBV. Women with HIV/HBV co-infection had significantly lower $\mathrm{CD} 4$ counts, highlighting the potential detriment of coinfection. However, in a small subset we did not find a difference in CD8+ $\mathrm{T}$ cell responses to HIV. These data contribute towards an improved understanding of the scale of the HIV/HBV coinfection problem in Africa, and suggest that adverse outcomes are mediated by factors other than CD8+ T cell responses to HIV.

\section{Authors' details}

${ }^{1}$ Nuffield Department of Medicine, University of Oxford, Oxford OX1 3SY, UK. ${ }^{2}$ Department of Infectious Diseases and Microbiology, Oxford University Hospitals, John Radcliffe Hospital, Headington, Oxford OX3 9DU, UK. ${ }^{3}$ Institute of Infection \& Global Health, University of Liverpool, Liverpool L69 7BE, UK. ${ }^{4}$ Department of Pediatrics, University of Oxford, Peter Medawar Building, Oxford OX1 3SY, UK. ${ }^{5}$ HIV Pathogenesis Program, Doris Duke Medical Research Institute, University of KwaZulu-Natal, Durban, South Africa. ${ }^{6} \mathrm{NIHR}$ Biomedical Research Center, John Radcliffe Hospital, Headington, Oxford OX3 9DU, UK.

Published: 23 May 2014

\section{doi:10.1186/1471-2334-14-S2-P18}

Cite this article as: Matthews et al:: Characterizing the epidemiology and interaction between HIV-1 and HBV co-infection in South Africa. BMC Infectious Diseases 2014 14(Suppl 2):P18.

${ }^{1}$ Nuffield Department of Medicine, University of Oxford, Oxford OX1 3SY, UK Full list of author information is available at the end of the article 